\title{
Linguistic Weighted Aggregation under Confidence Levels
}

\author{
Chonghui Zhang, ${ }^{1}$ Weihua Su, ${ }^{2,3}$ Shouzhen Zeng, ${ }^{2,4}$ and Linyun Zhang ${ }^{5}$ \\ ${ }^{1}$ College of Statistics and Mathematics, Zhejiang Gongshang University, Hangzhou 310018, China \\ ${ }^{2}$ College of Mathematics and Statistics, Zhejiang University of Finance and Economics, Hangzhou 310018, China \\ ${ }^{3}$ Center for Applied Statistics, Renmin University of China, Beijing 100872, China \\ ${ }^{4}$ College of Computer and Information, Zhejiang Wanli University, Ningbo 310015, China \\ ${ }^{5}$ Research Institute of Economic and Social Development, Zhejiang University of Finance and Economics, Hangzhou 310018, China \\ Correspondence should be addressed to Shouzhen Zeng; zszzxl@163.com
}

Received 8 July 2014; Revised 17 December 2014; Accepted 21 December 2014

Academic Editor: Kalyana C. Veluvolu

Copyright (C) 2015 Chonghui Zhang et al. This is an open access article distributed under the Creative Commons Attribution License, which permits unrestricted use, distribution, and reproduction in any medium, provided the original work is properly cited.

\begin{abstract}
We develop some new linguistic aggregation operators based on confidence levels. Firstly, we introduce the confidence linguistic weighted averaging (CLWA) operator and the confidence linguistic ordered weighted averaging (CLOWA) operator. These two new linguistic aggregation operators are able to consider the confidence level of the aggregated arguments provided by the information providers. We also study some of their properties. Then, based on the generalized means, we introduce the confidence generalized linguistic ordered weighted averaging (CGLOWA) operator. The main advantage of the CGLOWA operator is that it includes a wide range of special cases such as the CLOWA operator, the confidence linguistic ordered weighted quadratic averaging (CLOWQA) operator, and the confidence linguistic ordered weighted geometric (CLOWG) operator. Finally, we develop an application of the new approach in a multicriteria decision-making under linguistic environment and illustrate it with a numerical example.
\end{abstract}

\section{Introduction}

Information aggregation is a technique that analyzes the information in order to provide a final result. A variety of aggregation operators have been developed in the past few decades. One of the most common aggregation operators is the weighted average (WA) operator [1]. Another interesting one is the ordered weighted averaging (OWA) operator, originally introduced by Yager [2]. Its main advantage is that it provides a parameterized family of aggregation operators between the minimum and the maximum [3]. Since their introduction, many new extensions of the WA and OWA operator have been proposed, such as the weighted OWA (WOWA) operator [4], the OWA weighted averaging (OWAWA) operator [5], the induced ordered weighted averaging (IOWA) operator [6], the generalized OWA (GOWA) operator [7], the induced generalized OWA (IGOWA) operator [8], the power ordered weighted average (POWA) operator [9], and the continuous generalized ordered weighted averaging (CGOWA) operator [10].
Due to time pressure and decisions maker's limited expertise related with the problem domain, decision information about alternatives is often uncertain or fuzzy. As a result, it is more suitable to provide preferences by using linguistic variables rather than numerical ones. The use of the linguistic variable provides a direct way to assess vague environments where the information is provided by using expressions such as low, high, good, or bad $[11,12]$. In order to aggregate the linguistic information, many linguistic aggregation operators have been developed, such as the linguistic weighted ordered weighted averaging (LOWA) operator [13, 14], linguistic ordered weighted geometric averaging (LOWGA) operator [15], the induced linguistic generalized ordered weighted averaging (ILGOWA) operator [16], the linguistic generalized ordered weighted averaging (LGOWA) operator [17], and the linguistic generalized power ordered weighted average (LGPOWA) operator [18]. Recently, some new aggregation operators are developed to aggregate linguistic information. For example, Zhou and Chen [19] developed the induced linguistic continuous ordered weighted geometric (ILCOWG) 
operator, which is very suitable for group decision-making (GDM) problems taking the form of uncertain multiplicative linguistic preference relations. Wei et al. [20] developed a new aggregation operator called the belief structure generalized linguistic hybrid averaging (BS-GLHA) operator. Wang et al. [21] developed some linguistic cloud aggregation operators including the cloud weighted arithmetic averaging (CWAA) operator, cloud ordered weighted arithmetic averaging (COWA) operator, and cloud hybrid arithmetic (CHA) operator. Based on probabilistic information and induced aggregation operators, Merigó et al. [22] developed the induced linguistic probabilistic ordered weighted average (ILPOWA), which uses probabilities and OWA operators in the same formulation considering the degree of importance that each concept has in the formulation.

Most of the existing linguistic aggregation operators do not consider the confidence level of the aggregated arguments provided by the decision makers. However, in many real decision-making problems, such as the blind peer review of doctoral dissertation in China, the evaluation experts are requested to provide two types of information such as the performance of the evaluation objects and the familiarity with the evaluation areas (called confidence levels) [23, 24]. To overcome this issue, Yu [24] and Xia et al. [23] developed some induced aggregation operators under confidence levels, which can take into account the confidence levels of the aggregated arguments, while after reviewing the existing literature, it seems that there is no investigation on linguistic information aggregation under belief levels, which is an interesting and important issue. In this paper, we focus on the linguistic information aggregation issue in the situation where the confidences levels of the aggregated arguments are asked to be considered, and we develop a series of linguistic aggregation operators considering the confidence levels of the aggregated arguments, such as the confidence linguistic weighted averaging (CLWA) operator, the confidence linguistic ordered weighted averaging (CLOWA) operator, and the confidence generalized linguistic ordered weighted averaging (CGLOWA) operator. We also apply the developed operators to decision-making with linguistic information. Finally, an illustrative example has been given to show the developed method.

This paper is organized as follows. First, we briefly review some basic concepts such as the linguistic approach to be used throughout the paper, the LWA, and the LOWA operator. Second, we present the CLWA, CLOWA, and CGLOWA operator. Third, we discuss the applicability of the CGLOWA operator with a multicriteria decision-making example and we end the paper summarizing the main conclusions.

\section{Preliminaries}

This section briefly reviews the linguistic approach, the linguistic weighted average, and the linguistic OWA operator.

2.1. Linguistic Approach. The linguistic approach is an approximate technique, which represents qualitative aspects as linguistic values by means of linguistic variables. For computational convenience, let $S=\left\{s_{\alpha} \mid \alpha=1,2, \ldots, t\right\}$ be a finite and totally ordered discrete term set, where $s_{\alpha}$ represents a possible value for a linguistic variable. For example, a set of nine terms $S$ could be given as follows:

$$
\begin{gathered}
S=\left\{s_{1}=\mathrm{EL}, s_{2}=\mathrm{VL}, s_{3}=\mathrm{L}, s_{4}=\mathrm{LM}, s_{5}=\mathrm{M},\right. \\
\left.s_{6}=\mathrm{MH}, s_{7}=\mathrm{H}, s_{8}=\mathrm{VH}, s_{9}=\mathrm{EH}\right\} .
\end{gathered}
$$

Note that EL means extremely low, VL very low, L low, LM low-medium, $\mathrm{M}$ medium, $\mathrm{MH}$ medium-high, $\mathrm{H}$ high, $\mathrm{VH}$ very high, and $\mathrm{EH}$ extremely high. Usually, in these cases, it is required that in the linguistic term set there exist the following:

(i) a negation operator: $\operatorname{Neg}\left(s_{i}\right)=s_{j}$ such that $j=g+1-i$;

(ii) the set ordered $s_{i} \leq s_{j}$ if and only if $i \leq j$;

(iii) Max operator: $\operatorname{Max}\left(s_{i}, s_{j}\right)=s_{i}$ if $s_{i} \geq s_{j}$;

(iv) Min operator: $\operatorname{Min}\left(s_{i}, s_{j}\right)=s_{i}$ if $s_{i} \leq s_{j}$.

In order to preserve all the given information, $\mathrm{Xu}$ [25] extended the discrete term set $S$ to a continuous term set $\widehat{S}=\left\{s_{\alpha} \mid \alpha \in[1, t]\right\}$, where if $s_{\alpha} \in S$, then we call $s_{\alpha}$ the original term, and otherwise, we call $s_{\alpha}$ the virtual term. In general, the decision maker uses the original linguistic terms to evaluate alternatives, and the virtual linguistic terms can only appear in the actual calculation [26].

Consider any two linguistic terms $s_{\alpha}, s_{\beta} \in \widehat{S}$ and $\mu>0$; we define some operational laws as follows:

(i) $\mu s_{\alpha}=s_{\mu \alpha}$;

(ii) $s_{\alpha}+s_{\beta}=s_{\beta}+s_{\alpha}=s_{\alpha+\beta}$;

(iii) $\left(s_{\alpha}\right)^{\mu}=s_{\alpha^{\mu}}$;

(iv) $s_{\alpha} \times s_{\beta}=s_{\beta} \times s_{\alpha}=s_{\alpha \beta}$.

Note that this model is very useful for computing with words because it is very easy to use and it follows a similar methodology as the numerical information.

2.2. The Linguistic Weighted Average. The linguistic weighted average (LWA) [14] is a linguistic aggregation operator that uses the weighted average under uncertain environments assessed with linguistic information. It is defined as follows.

Definition 1. A LWA operator of dimension $n$ is a mapping LWA: $\widehat{S}^{n} \rightarrow \widehat{S}$, which has an associated weighting vector $W$ with $w_{i} \in[0,1]$ and $\sum_{i=1}^{n} w_{i}=1$, such that

$$
\operatorname{LWA}\left(s_{\alpha_{1}}, s_{\alpha_{2}}, \ldots, s_{\alpha_{n}}\right)=\sum_{i=1}^{n} w_{i} s_{\alpha_{i}} .
$$

2.3. The Linguistic OWA Operator. The OWA operator is a nonlinear operator, and an important feature is the reordering step. It has been studied in many documents and it has a wide range of applications [27-38]. By extending the OWA operator assessed with linguistic information, the LOWA operator can be defined as follows. 
Definition 2. A LOWA operator of dimension $n$ is a mapping LOWA: $\widehat{S}^{n} \rightarrow \widehat{S}$, which has an associated weighting vector $W$ with $w_{j} \in[0,1]$ and $\sum_{j=1}^{n} w_{j}=1$, such that

$$
\operatorname{LOWA}\left(s_{\alpha_{1}}, s_{\alpha_{2}}, \ldots, s_{\alpha_{n}}\right)=\sum_{j=1}^{n} w_{j} s_{\beta_{j}},
$$

where $s_{\beta_{j}}$ is the $j$ th largest of the $s_{\alpha_{i}}$.

The LOWA operator has been also extended under different frameworks including the use of Dempster-Shafer theory [20,39], induced aggregation operators [16, 26, 40], generalized aggregation operators $[17,41]$, distance measures [42], and power aggregation operators [18]. However, most of the existing linguistic aggregation operators do not consider the confidence level of the aggregated arguments provided by the information providers. Therefore, in the next section, we will develop some new linguistic aggregation operators, which can take into account the confidence levels of the aggregated arguments.

\section{Linguistic Information Aggregation Operators under Confidence Levels}

In some real decision-making problems, the evaluation experts are requested to provide two types of information such as the performance of the evaluation objects and the familiarity with the evaluation areas (called confidence levels). In this Section, we investigate the linguistic information aggregation methods under confidence levels and proposed a series of new linguistic aggregation operators.

3.1. Confidence Linguistic Weighted Averaging (CLWA) Operator. In the following, we propose the confidence linguistic weighted averaging (CLWA) operator and study the desirable properties of the proposed operator. The definition of the CLWA operator is given as follows.

Definition 3. A CLWA operator of dimension $n$ is a mapping CLWA: $R^{n} \times \widehat{S}^{n} \rightarrow \widehat{S}$, which has an associated weighting vector $W$ with $w_{i} \in[0,1]$ and $\sum_{i=1}^{n} w_{i}=1$, such that

$$
\operatorname{CLWA}\left(\left\langle l_{1}, s_{\alpha_{1}}\right\rangle,\left\langle l_{2}, s_{\alpha_{2}}\right\rangle, \ldots,\left\langle l_{n}, s_{\alpha_{n}}\right\rangle\right)=\sum_{i=1}^{n} w_{i}\left(l_{i} s_{\alpha_{i}}\right) \text {, }
$$

where $l_{i}$ is the belief levels of linguistic variable $s_{\alpha_{i}}, 0 \leq l_{i} \leq 1$. In particular, if $l_{1}=l_{2}=\cdots=l_{n}=1$, then the CLWA operator reduces to the LWA operator.

In the following example, we present a simple numerical example showing how to use the CLWA operator in an aggregation process.

Example 4. Assume the following arguments in an aggregation process: $A=\left(s_{1}, s_{4}, s_{3}, s_{7}\right)$ with the following confidence levels vector being $(0.6,0.5,0.9,0.6)$ and the following weighting vector $W=(0.3,0.2,0.1,0.4)$; then

$$
\operatorname{CLWA}\left(\left\langle 0.6, s_{1}\right\rangle,\left\langle 0.5, s_{4}\right\rangle,\left\langle 0.9, s_{3}\right\rangle,\left\langle 0.6, s_{7}\right\rangle\right)=s_{2.53} \text {. }
$$

Let $s_{\alpha_{i}}$ and $s_{\beta_{i}}(i=1,2, \ldots, n)$ be two collections of linguistic variables and $l_{i}\left(0 \leq l_{i} \leq 1\right)$ and $w_{i}\left(0 \leq w_{i} \leq 1\right)$ the confidence levels and weight of them, respectively, with $\sum_{i=1}^{n} w_{i}=1$. Then we can easily prove the CLWA operator has the properties of monotonicity, boundness, and idempotency as follows.

(1) (Monotonicity) If $s_{\alpha_{i}} \leq s_{\beta_{i}}$ for all $i$, then

$$
\begin{aligned}
\operatorname{CLWA} & \left(\left\langle l_{1}, s_{\alpha_{1}}\right\rangle, \ldots,\left\langle l_{n}, s_{\alpha_{n}}\right\rangle\right) \\
\leq \operatorname{CLWA} & \left(\left\langle l_{1}, s_{\beta_{1}}\right\rangle, \ldots,\left\langle l_{n}, s_{\beta_{n}}\right\rangle\right) .
\end{aligned}
$$

(2) (Boundness) Consider

$$
\min _{i}\left(l_{i} s_{\alpha_{i}}\right) \leq \operatorname{CLWA}\left(\left\langle l_{1}, s_{\alpha_{1}}\right\rangle, \ldots,\left\langle l_{n}, s_{\alpha_{n}}\right\rangle\right) \leq \max _{i}\left(l_{i} s_{\alpha_{i}}\right) \text {. }
$$

(3) (Idempotency) If $s_{\alpha_{i}}=s_{\alpha}$ for all $i$, then

$$
\operatorname{CLWA}\left(\left\langle l_{1}, s_{\alpha_{1}}\right\rangle, \ldots,\left\langle l_{n}, s_{\alpha_{n}}\right\rangle\right)=s_{\alpha} .
$$

3.2. Confidence Linguistic Ordered Weighted Aggregation (CLOWA) Operator. In this section, we introduce the idea of OWA [2] into confidence linguistic information aggregation problem and propose the confidence linguistic ordered weighted averaging (CLOWA) operator.

Definition 5. Let $\left(s_{\alpha_{1}}, s_{\alpha_{2}}, \ldots, s_{\alpha_{n}}\right)$ be a collection of linguistic sets and $l_{i}\left(0 \leq l_{i} \leq 1\right)$ the confidence levels linguistic variable $s_{\alpha_{i}}$. A CLOWA operator of dimension $n$ is a mapping CLOWA: $R^{n} \times \widehat{S}^{n} \rightarrow \widehat{S}$, which has an associated weighting vector $W$ with $w_{j} \in[0,1]$ and $\sum_{j=1}^{n} w_{j}=1$, such that

$$
\operatorname{CLOWA}\left(\left\langle l_{1}, s_{\alpha_{1}}\right\rangle,\left\langle l_{2}, s_{\alpha_{2}}\right\rangle, \ldots,\left\langle l_{n}, s_{\alpha_{n}}\right\rangle\right)=\sum_{i=1}^{n} w_{j}\left(l_{j} s_{\alpha_{j}}\right)
$$

where $l_{j} s_{\alpha_{j}}$ is the $j$ th largest of the $l_{i} s_{\alpha_{i}}$. In particular, if $l_{1}=$ $l_{2}=\cdots=l_{n}=1$, then the CLOWA operator reduces to the LOWA operator.

Example 6. Assume the following arguments in an aggregation process: $A=\left(s_{1}, s_{4}, s_{3}, s_{7}\right)$ with the following confidence levels vector being $(0.6,0.5,0.9,0.6)$ and the following weighting vector $W=(0.3,0.2,0.1,0.4)$; then

$$
\operatorname{CLOWA}\left(\left\langle 0.6, s_{1}\right\rangle,\left\langle 0.5, s_{4}\right\rangle,\left\langle 0.9, s_{3}\right\rangle,\left\langle 0.6, s_{7}\right\rangle\right)=s_{2.24} \text {. }
$$

The CLOWA operator is a mean or averaging operator. This is a reflection of the fact that the operator is monotonic, idempotent, bounded, and commutative. These properties are proven in the following theorems.

Theorem 7 (monotonicity). Let $f$ be the CLOWA operator; if $s_{\alpha_{i}} \leq s_{\beta_{i}}$ for all $i$, then

$$
f\left(\left\langle l_{1}, s_{\alpha_{1}}\right\rangle, \ldots,\left\langle l_{n}, s_{\alpha_{n}}\right\rangle\right) \leq f\left(\left\langle l_{1}, s_{\beta_{1}}\right\rangle, \ldots,\left\langle l_{n}, s_{\beta_{n}}\right\rangle\right) .
$$


Theorem 8 (idempotency). Let $f$ be the CLOWA operator; $s_{\alpha_{i}}=s_{\alpha}$ for all $i$; then

$$
f\left(\left\langle l_{1}, s_{\alpha_{1}}\right\rangle, \ldots,\left\langle l_{n}, s_{\alpha_{n}}\right\rangle\right)=s_{\alpha} .
$$

Theorem 9 (bounded). Let $f$ be the CLOWA operator; then

$$
\begin{aligned}
\min _{i}\left(l_{i} s_{\alpha_{i}}\right) & \leq \operatorname{CLOWA}\left(\left\langle l_{1}, s_{\alpha_{1}}\right\rangle, \ldots,\left\langle l_{n}, s_{\alpha_{n}}\right\rangle\right) \\
& \leq \max _{i}\left(l_{i} s_{\alpha_{i}}\right) .
\end{aligned}
$$

Theorem 10 (commutativity). Let $f$ be the CLOWA operator. Then

$$
f\left(\left\langle l_{1}, s_{\alpha_{1}}\right\rangle, \ldots,\left\langle l_{n}, s_{\alpha_{n}}\right\rangle\right)=f\left(\left\langle l_{1}^{\prime}, s_{\alpha_{1}}^{\prime}\right\rangle, \ldots,\left\langle l_{n}^{\prime}, s_{\alpha_{n}}^{\prime}\right\rangle\right),
$$

where $\left(\left\langle l_{1}^{\prime}, s_{\alpha_{1}}^{\prime}\right\rangle, \ldots,\left\langle l_{n}^{\prime}, s_{\alpha_{n}}^{\prime}\right\rangle\right)$ is any permutation of the arguments $\left(\left\langle l_{1}, s_{\alpha_{1}}\right\rangle, \ldots,\left\langle l_{n}, s_{\alpha_{n}}\right\rangle\right)$. Note that the CLWA operator does not have this property.

3.3. Confidence Generalized Linguistic Ordered Weighted Averaging (CGLOWA) Operator. The confidence generalized linguistic ordered weighted averaging (CGLOWA) operator is an extension of the OWA operator that uses the main characteristics of both the CLOWA and the GOWA operator. Then, we can obtain a generalization that includes the CLOWA operator and many other situations, such as confidence linguistic ordered weighted geometric (CLOWG) operator and confidence linguistic ordered weighted harmonic averaging (CLOWHA) operator. It can be defined as follows.

Definition 11. Let $\left(s_{\alpha_{1}}, s_{\alpha_{2}}, \ldots, s_{\alpha_{n}}\right)$ be a collection of linguistic sets and $l_{i}\left(0 \leq l_{i} \leq 1\right)$ the confidence levels linguistic variable $s_{\alpha_{i}}$. A CGLOWA operator of dimension $n$ is a mapping CGLOWA: $R^{n} \times \widehat{S}^{n} \rightarrow \widehat{S}$, which has an associated weighting vector $W$ with $w_{j} \in[0,1]$ and $\sum_{j=1}^{n} w_{j}=1$, such that

$$
\begin{aligned}
& \operatorname{CGLOWA}\left(\left\langle l_{1}, s_{\alpha_{1}}\right\rangle,\left\langle l_{2}, s_{\alpha_{2}}\right\rangle, \ldots,\left\langle l_{n}, s_{\alpha_{n}}\right\rangle\right) \\
& =\left(\sum_{i=1}^{n} w_{j}\left(l_{j} s_{\alpha_{j}}\right)^{\lambda}\right)^{1 / \lambda},
\end{aligned}
$$

where $l_{j} s_{\alpha_{j}}$ is the $j$ th largest of the $l_{i} s_{\alpha_{i}}$ and $\lambda$ is a parameter such that $\lambda \in(-\infty, 0) \cup(0,+\infty)$. In particular, if $l_{1}=l_{2}=$ $\cdots=l_{n}=1$, then the CGLOWA operator reduces to the GLOWA operator [17].

Example 12. Assume the following arguments in an aggregation process: $A=\left(s_{-2}, s_{0}, s_{3}, s_{-1}\right)$ with the following confidence levels vector being $(0.6,0.5,0.9,0.6)$ and the following weighting vector $W=(0.3,0.2,0.1,0.4)$. And without loss of generality, let $\lambda=2$; then

$$
\operatorname{CGLOWA}\left(\left\langle 0.6, s_{1}\right\rangle,\left\langle 0.5, s_{4}\right\rangle,\left\langle 0.9, s_{3}\right\rangle,\left\langle 0.6, s_{7}\right\rangle\right)=s_{2.70} .
$$

Note that the different aggregated results can be obtained if the parameter $\lambda$ takes different values. The selection of the particular parameter $\lambda$ depends on the particular interest of the decision maker in the specific problem considered.

Similar to the CLOWA operator, the CGLOWA operator is also monotonic, idempotent, bounded, and commutative. If we analyze different values of the parameter $\lambda$, we obtain a group of particular cases. For example, we have the following.

(i) If $\lambda=1$, the CGLOWA operator becomes the CLOWA operator.

(ii) If $\lambda=2$, we get the confidence linguistic ordered weighted quadratic averaging (CLOWQA) operator.

(iii) If $\lambda \rightarrow 0$, we form the confidence linguistic ordered weighted geometric (CLOWG) operator

(iv) If $\lambda=-1$, the confidence linguistic ordered weighted harmonic averaging (CLOWHA) operator is obtained.

\section{An Application of the Proposed Operator to Multicriteria Decision-Making}

The CGLOWA (or CLOWA) operator is applicable in a wide range of situations, such as decision-making, statistics, engineering, and economics. In summary, all of the studies that use the OWA operator can be revised and extended by using this new approach.

In the following, we are going to develop a decisionmaking method about the use of the CGLOWA in a multicriteria decision-making problem. For a multicriteria decisionmaking problem, let $X=\left\{X_{1}, X_{2}, \ldots, X_{m}\right\}$ be a discrete set of alternatives and let $G=\left\{G_{1}, G_{2}, \ldots, G_{n}\right\}$ be the set of criteria. The main steps of the decision-making methods are as follows.

Step 1. The decision makers provide their evaluations and belief levels about the alternative $X_{i}$ under the attribute $G_{j}$, forming the decision matrix $D=\left(a_{i j}\right)_{m \times n}$, where $a_{i j} \in S$ is a preference value, which takes the form of linguistic variable.

Step 2. The decision makers provide their belief levels of the evaluations expressed by $l_{i j}(i=1,2, \ldots, m ; j=1,2, \ldots, n)$, where $l_{i j}$ is the belief levels of linguistic variable $a_{i j}, i=$ $1,2, \ldots, m ; j=1,2, \ldots, n$.

Step 3. Utilize the CGLOWA (or some of its special cases) operator to aggregate the evaluations $a_{i j}$ for each alternative $X_{i}$. Consider

$$
a_{i}=\operatorname{CGLOWA}\left(\left\langle l_{i 1}, a_{i 1}\right\rangle, \ldots,\left\langle l_{i n}, a_{i n}\right\rangle\right), \quad i=1,2, \ldots, m .
$$

Step 4. Rank all the alternatives $X_{i}(i=1,2, \ldots, m)$ and select the best one $(s)$ in accordance with $a_{i}(i=1,2, \ldots, m)$.

Example 13. Let us consider a blind peer review of doctoral dissertation evaluation problem in China's university (adapted from [24]). In many Chinese universities, the doctoral dissertation will be reviewed by several experts anonymously. And they will review dissertation according to 
TABLE 1: Linguistic fuzzy decision making metric.

\begin{tabular}{lccccc}
\hline & $G_{1}$ & $G_{2}$ & $G_{3}$ & $G_{4}$ & $G_{5}$ \\
\hline$X_{1}$ & $s_{3}$ & $s_{5}$ & $s_{4}$ & $s_{7}$ & $s_{9}$ \\
$X_{2}$ & $s_{5}$ & $s_{6}$ & $s_{6}$ & $s_{7}$ & $s_{8}$ \\
$X_{3}$ & $s_{2}$ & $s_{4}$ & $s_{7}$ & $s_{8}$ & $s_{7}$ \\
$X_{4}$ & $s_{4}$ & $s_{7}$ & $s_{5}$ & $s_{6}$ & $s_{6}$ \\
$X_{5}$ & $s_{3}$ & $s_{8}$ & $s_{7}$ & $s_{6}$ & $s_{9}$ \\
\hline
\end{tabular}

five criteria, including topic selection and literature review $\left(G_{1}\right)$, innovation $\left(G_{2}\right)$, theory basis and special knowledge $\left(G_{3}\right)$, capacity of scientific research $\left(G_{4}\right)$, and theses writing $\left(G_{5}\right)$. Unlike many existing evaluation methods, the experts not only required to provide the evaluation results of the doctoral dissertation but also asked to give the degrees to which they are familiar with the research topics (called belief levels). Suppose there are five declarations that need to be reviewed by expert; the degree of familiarity of the five declarations provided by expert is $l_{j}=(0.8,0.7,0.9,0.8,0.6)$. Suppose the experts give the decision matrix under a linguistic framework of nine linguistic terms in the set as explained in Section 2.1, shown in Table 1.

Suppose that the weighting vector associating with the CGLOWA operator is $W=(0.11,0.24,0.30,0.24,0.11)$, which is derived by using the normal distribution based method [1]. With this information, it is possible to aggregate the available information in order to take a decision. By using some key particular cases of the CGLOWA operators to aggregate the linguistic variables for five declarations, the aggregated results and the ranking of the declarations are shown in Table 2.

As we can see, depending on the aggregation operator used, the results and decisions may be different. Therefore, the decision about which declarations to select may be also different. Note that, in this example, the optimal choice is the same for all aggregation operators.

If we do not consider the confidence levels factor, in other words, all the criteria of the evaluated objects are treated with sure familiar by the decision maker, then our proposed CGLOWA operator is reduced to the existing GLOWA operator [17]. The aggregated results of this example by the types of the GLOWA operators, such as linguistic OWA (LOWA) operator, the linguistic OWG (LOWG) operator, the linguistic OWHA (LOWHA) operator, and the linguistic ordered weighted quadratic averaging (LOWQA) operator, are listed in Table 3.

It can be easily seen that the ranking of the five doctoral dissertations obtained by the proposed CLGOWA operator is similar to the result by the LGOWA operator. However, in real-life decision process, the decision maker(s) may not be familiar with the doctoral dissertation absolutely. To deal with such situations, the proposed CLGOWA operator is useful tool. From the above analysis, we can see that the main advantage of using the CGLOWA operator is that it can consider the belief levels of the decision maker(s). Another main advantage is that it includes a wide range of particular cases such as the CLOWA, the CLOWQA, and the CLOWG operator. Due to the fact that each particular family of CGLOWA operator may give different results, the decision maker(s) will select for his decision the one that is closest to his interests.

\section{Concluding Remarks}

In this paper, we have developed some new linguistic information aggregation operators by introducing the belief levels. The confidence linguistic weighted averaging (CLWA) operator, the confidence linguistic ordered weighted averaging (CLOWA) operator, and the confidence generalized linguistic ordered weighted averaging (CGLOWA) operator have been introduced. We have studied various properties of the developed operators. We have also presented an application of the CGLOWA operator to a multicriteria decision-making problem concerning the dissertation evaluation problem. We have seen that the CGLOWA is very useful because it represents very well the uncertain information by using linguistic labels, as well as considering the belief levels of the decision makers. Moreover, it includes a wide range of particular cases such as the CLOWA, the CLOWQA, and the CLOWG operator. Due to the fact that each particular family of CGLOWA operator may give different results, the decision maker gets a more complete view of the decision problem and is able to select the alternative that is closest to his interests.

It is worth pointing out that we can extend the confidence aggregation, using a similar method, to deal with the other fuzzy situations where the arguments are expressed in interval values or triangular fuzzy values. In future research, we expect to develop further extensions by adding new characteristics in the problem such as the use of inducing variables or probabilistic aggregations.

\section{Conflict of Interests}

The authors declare that there is no conflict of interests regarding the publication of this paper.

\section{Acknowledgments}

The authors are particularly grateful to the editor and anonymous reviewers who gave useful suggestions and considerably helped in improving the paper. This paper is supported by the MOE Project of Humanities and Social Sciences (nos. 14YJC910006, 13JJD910002), Zhejiang Province Natural Science Foundation (no. LQ14G010002), Statistical Scientific Key Research Project of China (no. 2013LZ48), Key Research Center of Philosophy and Social Science of Zhejiang Province and Modern Port Service Industry and Creative Culture Research Center, Zhejiang Provincial Key Research Base for Humanities and Social Science Research (Statistics), 
TABLE 2: Aggregated results by the CGLOWA and the rankings of alternatives.

\begin{tabular}{lcccccc}
\hline & $X_{1}$ & $X_{2}$ & $X_{3}$ & $X_{4}$ & $X_{5}$ & Ranking \\
\hline CLOWA $(\lambda=1)$ & $s_{4.10}$ & $s_{4.80}$ & $s_{4.32}$ & $s_{4.23}$ & $s_{5.24}$ & $X_{5}>X_{2}>X_{3}>X_{4}>X_{1}$ \\
CLOWQA $(\lambda=2)$ & $s_{4.23}$ & $s_{4.83}$ & $s_{4.64}$ & $s_{4.46}$ & $s_{5.37}$ & $X_{5}>X_{2}>X_{3}>X_{4}>X_{1}$ \\
CLOWG $(\lambda \rightarrow 0)$ & $s_{3.96}$ & $s_{4.77}$ & $s_{3.96}$ & $s_{4.39}$ & $s_{5.07}$ & $X_{5}>X_{2}>X_{4}>X_{1}=X_{3}$ \\
CLOWHA $(\lambda=-1)$ & $s_{3.82}$ & $s_{4.73}$ & $s_{3.56}$ & $s_{4.34}$ & $s_{4.84}$ & $X_{5}>X_{2}>X_{4}>X_{1}>X_{3}$ \\
\hline
\end{tabular}

TABLE 3: Aggregated results by the GLOWA operator and the rankings of alternatives.

\begin{tabular}{|c|c|c|c|c|c|c|}
\hline & $X_{1}$ & $X_{2}$ & $X_{3}$ & $X_{4}$ & $X_{5}$ & Ranking \\
\hline LOWA $(\lambda=1)$ & $s_{5.46}$ & $s_{6.35}$ & $s_{5.84}$ & $s_{5.56}$ & $s_{6.78}$ & $X_{5} \succ X_{2} \succ X_{3}>X_{4} \succ X_{1}$ \\
\hline LOWQA $(\lambda=2)$ & $s_{5.74}$ & $s_{6.40}$ & $s_{6.15}$ & $s_{5.71}$ & $s_{6.97}$ & $X_{5}>X_{2}>X_{3}>X_{1}>X_{4}$ \\
\hline LOWG $(\lambda \rightarrow 0)$ & $s_{5.18}$ & $s_{6.30}$ & $s_{5.41}$ & $s_{5.59}$ & $s_{6.52}$ & $X_{5}>X_{2}>X_{4}>X_{3}>X_{1}$ \\
\hline $\operatorname{LOWHA}(\lambda=-1)$ & $s_{4.92}$ & $s_{6.25}$ & $s_{4.86}$ & $s_{5.52}$ & $s_{6.18}$ & $X_{2}>X_{5}>X_{4}>X_{1}>X_{3}$ \\
\hline
\end{tabular}

Projects in Science and Technique of Ningbo Municipal (no. 2012B82003), and Ningbo Natural Science Foundation (no. 2013A610286).

\section{References}

[1] Z. S. Xu, "An overview of methods for determining OWA weights," International Journal of Intelligent Systems, vol. 20, no. 8, pp. 843-865, 2005.

[2] R. R. Yager, "On ordered weighted averaging aggregation operators in multicriteria decisionmaking," IEEE Transactions on Systems, Man, and Cybernetics B: Cybernetics, vol. 18, no. 1, pp. 183-190, 1988.

[3] J. M. Merigó and A. M. Gil-Lafuente, "New decision-making methods and their application in the selection of financial products," Information Sciences, vol. 180, pp. 2085-2094, 2010.

[4] V. Torra, "The weighted OWA operator," International Journal of Intelligent Systems, vol. 12, no. 2, pp. 153-166, 1997.

[5] J. M. Merigó, "A unified model between the weighted average and the induced OWA operator," Expert Systems with Applications, vol. 38, no. 9, pp. 11560-11572, 2011.

[6] R. R. Yager and D. P. Filev, "Induced ordered weighted averaging operators," IEEE Transactions on Systems, Man, and Cybernetics B: Cybernetics, vol. 29, no. 2, pp. 141-150, 1999.

[7] R. R. Yager, "Generalized OWA aggregation operators," Fuzzy Optimization and Decision Making, vol. 3, no. 1, pp. 93-107, 2004.

[8] J. M. Merigó and A. M. Gil-Lafuente, “The induced generalized OWA operator," Information Sciences, vol. 179, no. 6, pp. 729741, 2009.

[9] R. R. Yager, “The power average operator," IEEE Transactions on Systems, Man, and Cybernetics A: Systems and Humans, vol. 31, no. 6, pp. 724-731, 2001.

[10] L.-G. Zhou and H.-Y. Chen, "Continuous generalized OWA operator and its application to decision making," Fuzzy Sets and Systems, vol. 168, pp. 18-34, 2011.

[11] F. Herrera and E. Herrera-Viedma, "Linguistic decision analysis: steps for solving decision problems under linguistic information," Fuzzy Sets and Systems, vol. 115, no. 1, pp. 67-82, 2000.

[12] L. A. Zadeh, "The concept of a linguistic variable and its application to approximate reasoning-I," Information Sciences, vol. 8, no. 3, pp. 199-249, 1975.

[13] F. Herrera, E. Herrera-Viedma, and J. L. Verdegay, "A sequential selection process in group decision making with a linguistic assessment approach," Information Sciences, vol. 85, no. 4, pp. 223-239, 1995.

[14] Z. S. Xu, "EOWA and EOWG operators for aggregating linguistic labels based on linguistic preference relations," International Journal of Uncertainty, Fuzziness and Knowledge-Based Systems, vol. 12, no. 6, pp. 791-810, 2004.

[15] Z. S. Xu, "A method based on linguistic aggregation operators for group decision making with linguistic preference relations," Information Sciences, vol. 166, no. 1-4, pp. 19-30, 2004.

[16] J. M. Merigó, A. M. Gil-Lafuente, L.-G. Zhou, and H.-Y. Chen, "Induced and linguistic generalized aggregation operators and their application in linguistic group decision making," Group Decision and Negotiation, vol. 21, no. 4, pp. 531-549, 2012.

[17] J. M. Merigó, A. M. Gil-Lafuente, L. G. Zhou, and H. Y. Chen, "Generalization of the linguistic aggregation operator and its application in decision making," Journal of Systems Engineering and Electronics, vol. 22, no. 4, pp. 593-603, 2011.

[18] L. G. Zhou and H. Y. Chen, "A generalization of the power aggregation operators for linguistic environment and its application in group decision making," Knowledge-Based Systems, vol. 26, pp. 216-224, 2012.

[19] L. G. Zhou and H. Y. Chen, "The induced linguistic continuous ordered weighted geometric operator and its application to group decision making," Computers \& Industrial Engineering, vol. 66, pp. 222-232, 2013.

[20] G.-W. Wei, X. Zhao, and R. Lin, "Some hybrid aggregating operators in linguistic decision making with Dempster-Shafer belief structure," Computers \& Industrial Engineering, vol. 65, no. 4, pp. 646-651, 2013.

[21] J. Q. Wang, P. Lu, H. Y. Zhang, and X. H. Chen, "Method of multi-criteria group decision-making based on cloud aggregation operators with linguistic information," Information Sciences, vol. 274, pp. 177-191, 2014.

[22] J. M. Merigó, M. Casanovas, and P. Daniel, "Linguistic group decision making with induced aggregation operators and probabilistic information," Applied Soft Computing, vol. 24, pp. 669678, 2014.

[23] M. M. Xia, Z. S. Xu, and N. Chen, "Induced aggregation under confidence levels," International Journal of Uncertainty, Fuzziness and Knowledge-Based Systems, vol. 19, no. 2, pp. 201-227, 2011.

[24] D. J. Yu, "Intuitionistic fuzzy information aggregation under confidence levels," Applied Soft Computing Journal, vol. 19, pp. 147-160, 2014. 
[25] Z. S. Xu, "Deviation measures of linguistic preference relations in group decision making," Omega, vol. 33, no. 3, pp. 249-254, 2005.

[26] Z. S. Xu, "Induced uncertain linguistic OWA operators applied to group decision making," Information Fusion, vol. 7, no. 2, pp. 231-238, 2006.

[27] G. Beliakov, A. Pradera, and T. Calvo, Aggregation Functions: A Guide for Practitioners, Springer, Berlin, Germany, 2007.

[28] S. P. Wan, "2-Tuple linguistic hybrid arithmetic aggregation operators and application to multi-attribute group decision making," Knowledge-Based Systems, vol. 15, pp. 31-40, 2013.

[29] G. W. Wei, "Uncertain linguistic hybrid geometric mean operator and its application to group decision making under uncertain linguistic environment," International Journal of Uncertainty, Fuzziness, Knowledge-Based Systems, vol. 17, pp. 251-267, 2009.

[30] G. W. Wei and X. F. Zhao, "Some induced correlated aggregating operators with intuitionistic fuzzy information and their application to multiple attribute group decision making," Expert Systems with Applications, vol. 39, no. 2, pp. 2026-2034, 2012.

[31] Y. J. Xu and H. M. Wang, "The induced generalized aggregation operators for intuitionistic fuzzy sets and their application in group decision making," Applied Soft Computing Journal, vol. 12, no. 3, pp. 1168-1179, 2012.

[32] X. H. Yu and Z. S. Xu, "Prioritized intuitionistic fuzzy aggregation operators," Information Fusion, vol. 14, pp. 108-116, 2013.

[33] D. J. Yu, "Intuitionistic fuzzy geometric Heronian mean aggregation operators," Applied Soft Computing Journal, vol. 13, no. 2, pp. 1235-1246, 2013.

[34] R. R. Yager, J. Kacprzyk, and G. Beliakov, Recent Developments on the Ordered Weighted Averaging Operators: Theory and Practice, Springer, Berlin, Germany, 2011.

[35] S. Z. Zeng, "Some intuitionistic fuzzy weighted distance measures and their application to group decision making," Group Decision and Negotiation, vol. 22, no. 2, pp. 281-298, 2013.

[36] S. Z. Zeng, J. M. Merigó, and W. H. Su, "The uncertain probabilistic OWA distance operator and its application in group decision making," Applied Mathematical Modelling, vol. 37, no. 9, pp. 6266-6275, 2013.

[37] H. M. Zhang, "The multiattribute group decision making method based on aggregation operators with interval-valued 2-tuple linguistic information," Mathematical and Computer Modelling, vol. 56, no. 1-2, pp. 27-35, 2012.

[38] H. M. Zhang, "Some interval-valued 2-tuple linguistic aggregation operators and application in multiattribute group decision making," Applied Mathematical Modelling, vol. 37, no. 6, pp. 4269-4282, 2013.

[39] J. M. Merigó, M. Casanovas, and L. Martínez, "Linguistic aggregation operators for linguistic decision making based on the Dempster-Shafer theory of evidence," International Journal of Uncertainty, Fuzziness and Knowledge-Based Systems, vol. 18, no. 3, pp. 287-304, 2010.

[40] J. Lan, Q. Sun, Q. Chen, and Z. Wang, "Group decision making based on induced uncertain linguistic OWA operators," Decision Support Systems, vol. 55, no. 1, pp. 296-303, 2013.

[41] B. Peng, C. Ye, and S. Z. Zeng, "Uncertain pure linguistic hybrid harmonic averaging operator and generalized interval aggregation operator based approach to group decision making," Knowledge-Based Systems, vol. 36, pp. 175-181, 2012.

[42] S. Z. Zeng and W. H. Su, "Linguistic induced generalized aggregation distance operators and their application to decision making," Economic Computer and Economic Cybernetics Studies and Research, vol. 46, pp. 155-172, 2012. 


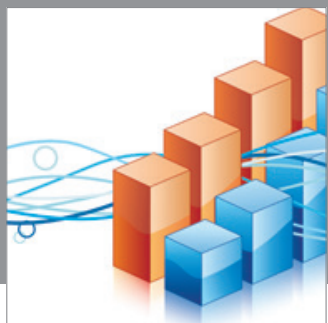

Advances in

Operations Research

mansans

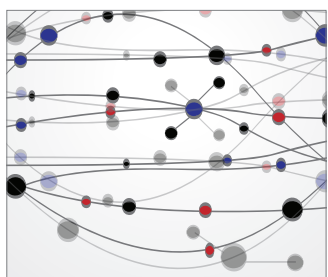

The Scientific World Journal
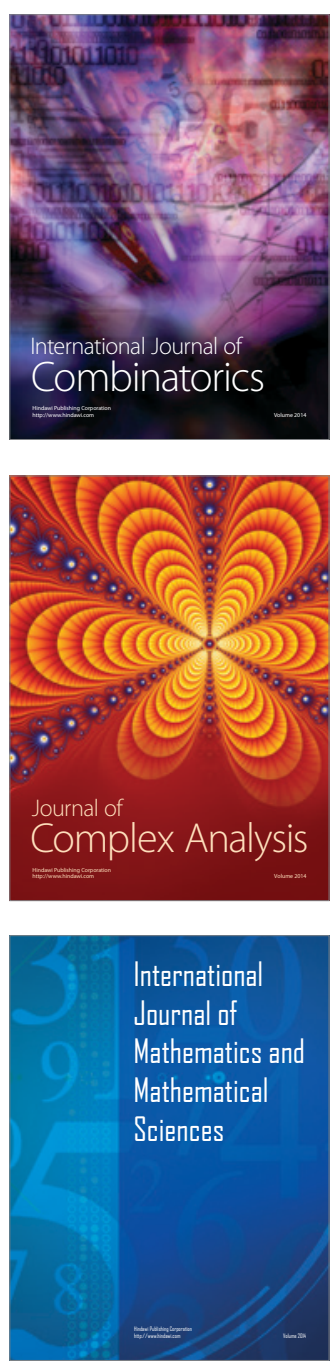
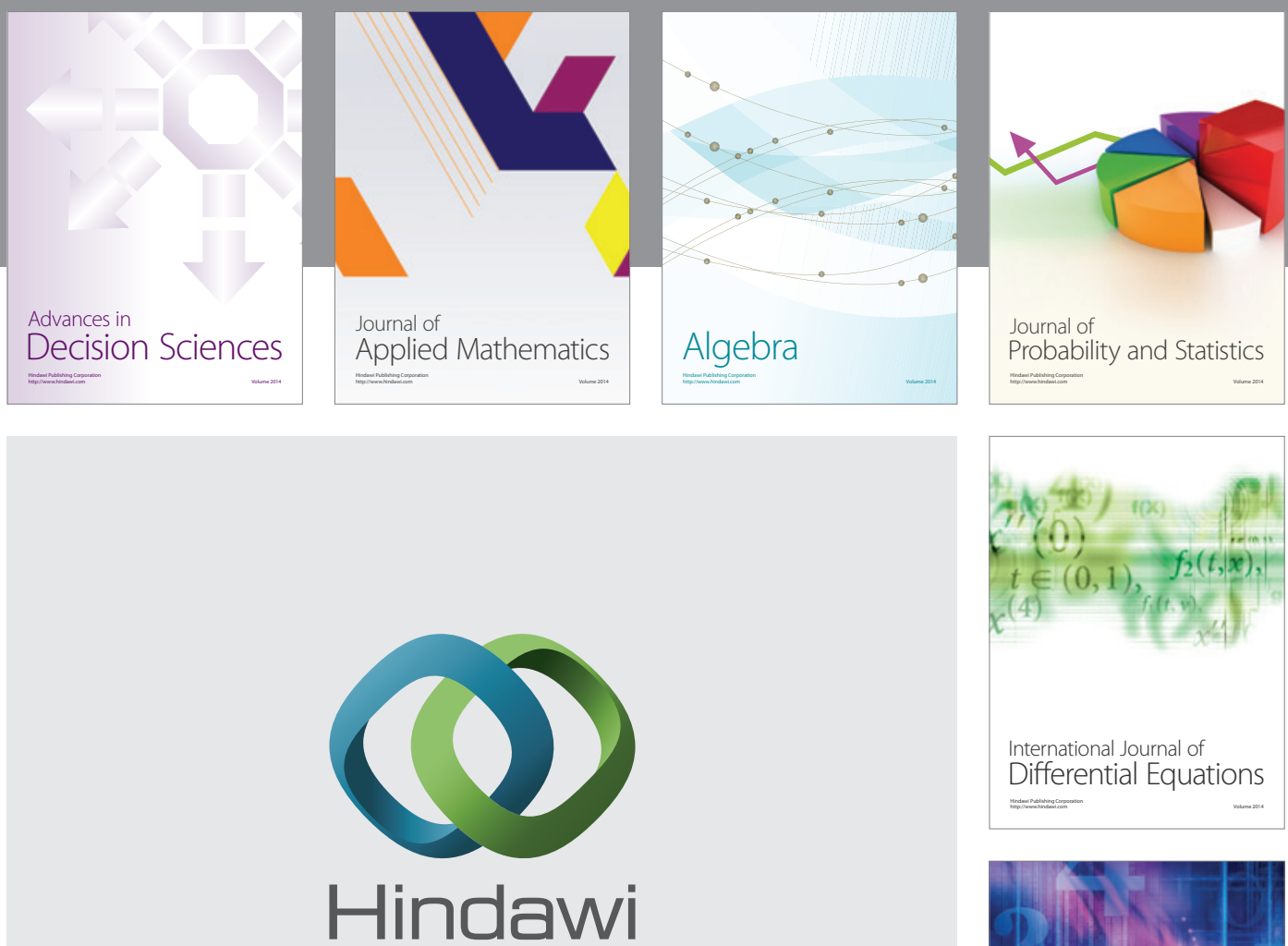

Submit your manuscripts at http://www.hindawi.com
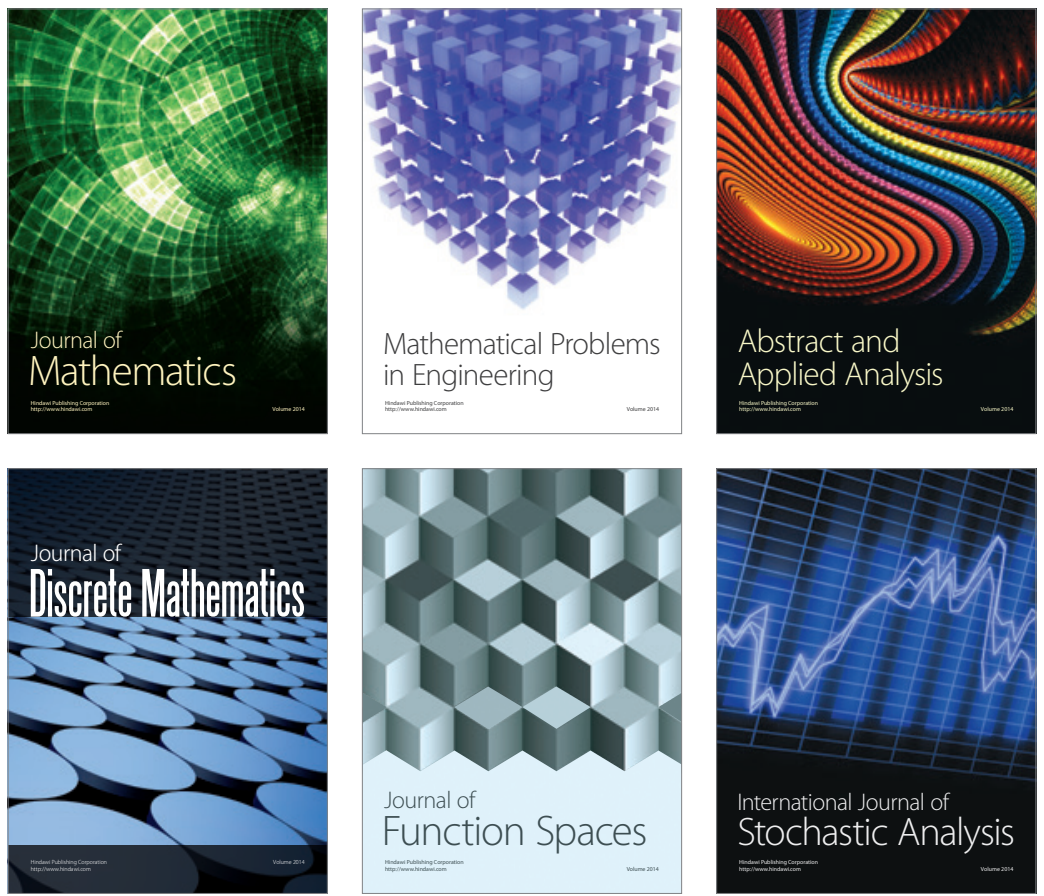

Journal of

Function Spaces

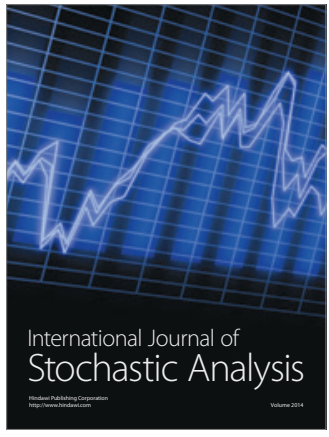

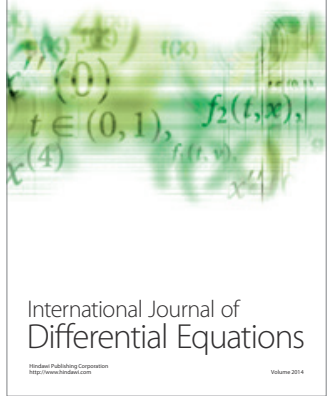
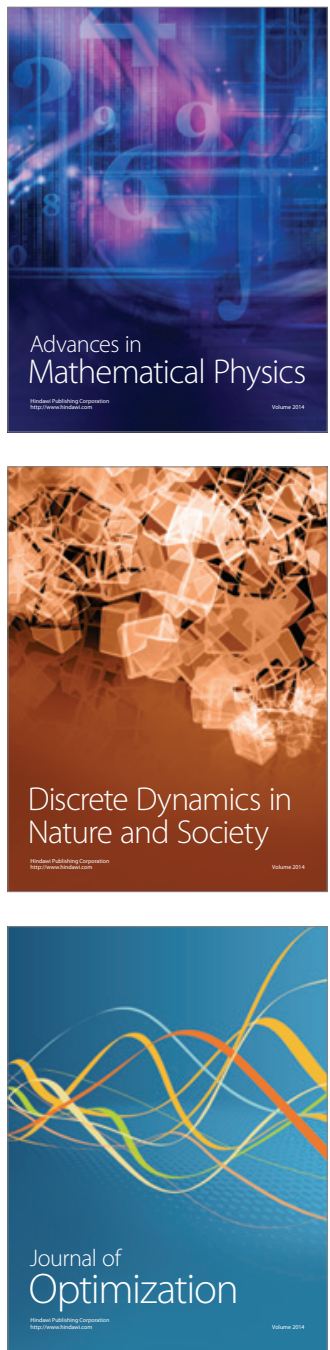